\title{
OR-4
}

\section{New Apicidins and a Polyketide from the Fungus Fusarium Semitectum}

\author{
Suciati $^{1,2, *}$ and Mary J. Garson ${ }^{1}$ \\ ${ }^{I}$ School of Chemistry and Molecular Biosciences, The University of Queensland, Brisbane, Qld 4072, Australia; \\ ${ }^{2}$ Faculty of Pharmacy, Airlangga University, Surabaya, East Java 60286, Indonesia; E-mail: suciati@uqconnect.edu.au
}

Chemical investigation of the culture of Fusarium semitectum isolated from a dead cicada skin collected in Indonesia has yielded three new tetrapeptides, namely apicidins E - G (1-3) together with the new polyketide 4 and six known compounds. The structures of the new metabolites were deduced by NMR and mass spectrometry, while the structure of polyketide $\mathbf{4}$, possessing an unusual eight-membered ring, was further confirmed by methylation to the methoxy derivative $\mathbf{5}$. Based on the result of this study together with earlier reports from the literature, we can suggest that tetrapeptide apicidin and its congeners are possibly chemotaxonomic markers of this fungal species.

Keywords: Fungus, Fusarium semitectum, tetrapeptides, apicidin, polyketide. 